Voix et Images

\title{
Pour saluer Jacques Ferron, ou Victor-Lévy Beaulieu, pèlerin et pénitent
}

\section{Robert Major}

Volume 17, numéro 1 (49), automne 1991

Louky Bersianik

URI : https://id.erudit.org/iderudit/200947ar

DOI : https://doi.org/10.7202/200947ar

Aller au sommaire du numéro

Éditeur(s)

Université du Québec à Montréal

ISSN

0318-9201 (imprimé)

1705-933X (numérique)

Découvrir la revue

Citer cet article

Major, R. (1991). Pour saluer Jacques Ferron, ou Victor-Lévy Beaulieu, pèlerin et pénitent. Voix et Images, 17(1), 132-137. https://doi.org/10.7202/200947ar d'utilisation que vous pouvez consulter en ligne.

https://apropos.erudit.org/fr/usagers/politique-dutilisation/ 
Essai

\section{Pour saluer Jacques Ferron, ou Victor-Lévy Beaulieu, pèlerin et pénitent.}

\section{Robert Major, Université d'Ottawa}

Ouvrant le dernier essai de. Victor-Lévy Beaulieu, Docteur Ferron, pèlerinage ${ }^{1}$, et avant même d'en commencer la lecture, à le soupeser et à le flairer, tout ainsi que le chien de Rabelais rencontrant quelqué os médullaire, me revient en mémoire le vif plaisir de lecture que m'avait procuré le premier des grands essais de cet auteur, Pour saluer Victor Hugo, publié en 1970. Un collègue français, plus âgé et plus savant, spécialiste de Victor Hugo de surcroît (et retourné en France depuis), avait beau m'assurer que cet essai était une fumisterie, nul pour un hugolien, rien n'y faisait. 'Je l'avais lu avec enthousiasme, je le défendais avec ardeur. Un écrivain québécois se colletait avec un des géants de la littérature universelle, prenait sa mesure, se l'assimilait, le rendait familier, le domestiquait littéralement en l'obligeant à vivre dans sa présence quotidienne et en se l'appropriant. Au point de se saisir de son prénom. Combat de Jacob et de l'ange dont je devinais confusément l'enjeu.

Il s'agissait, d'une part, d'une leçon sur le bon usage des géants littéraires qui, en publiant, et malgré leurs prétentions, ont accepté de se livrer pieds et poings liés à notre spoliation: à chacun des lecteurs de les exploiter à sa guise. Par ailleurs, il m'était évident aussi que Victor-Lévy Beaulieu n'était pas un lecteur ordinaire. Comme écrivain et par un procédé analogue à ce que Faguet appelait jadis l'innutrition (pour décrire la spoliation exercée par Ronsard à l'endroit de ses modèles), Victor-Ĺvey Beaulieu s'appropriait Victor Hugo pour composer sa propre œuvre; de plus, par ce choix premier d'un écrivain éponyme et fétiche, il se plaçait d'emblée dans la compagnie des plus grands. Volonté prométhéenne qui ne m'offusquait pas ni ne me paraissait prétentieuse. Normale au contraire, saine et réjouissante, pour une certaine catégorie d'écrivains dionysiaques. Autrement, pourquoi écrire?

Il y a vingt ans de cela, déjà ! Se sont succédé d'autres essais et d'autres appropriations de Victor-Lévy Beaulieu: Jack Kerouac, essai-poulet en 1972, et le superbe Monsieur Melville en 1978. Et tous ces romans, tous ces écrits de polygraphe qui ont fait de 
Beaulieu l'écrivain dominant de sa génération, et dont il ne saurait être question ici, car seul l'essayiste nous importe. Or voici justement que l'essayiste, plusieurs années après son périple américain (Kerouac et Melville), entame un pèlerinage québécois, à la recherche de son père en littérature, Jacques Ferron.

Mais sans doute ne devrais-je pas distinguer ainsi le romancier et l'essayiste, comme je viens de le faire. Ces frontières génériques sont instables, à plus forte raison chez Beaulieu, et ne tiennent guère devant la réalité du texte. On se souvient qu'au début de Monsieur Melville,' Abel Beauchemin, l'écrivain-narrateur de cet essai et l'alter ego fictif de Victor-Lévy Beaulieu, avait donné congé à sès personnages pendant deux ans, le temps de partir à la recherche de Herman Melville, auteur de Moby Dick. Vaine tentative de s'éloigner du roman ou d'éloigner le roman de soi ! «Lecture-fiction i: ainsi seront finalement identifiés les trois volumes de Monsieur Melville dans la liste des œuvres de l'auteur (page de garde), même si cet indicateur générique ne paraît pas sur la page de titre. Lecturefiction, en effet. Car l'essai pour Beaulieu est fiction tout autant qu'étude. Ainsi; les personnages romanesques sont congédiés en vain: ils sont tenaces et restent présents, pénétrant l'essai et s'y lovant. Et non seulement les personnages des œuvres antérieures (Père, l'hôte d'Abel pour le marathon d'écriture du Melville, Job J., Una, France...). Ces personnages ne veulent pas laisser l'essayiste en paix. Mais, de plus, apparaissent de nouveaux personnages, inédits, qui voudront ultérieurement trouver voix dans le cycle romanesque.

Ainsi en est-il de cette mystérieuse Samm, Montagnaise, qui surgit soudainement dans Monsieur Melville et qui deviendra accompagnatrice, âme sœur, confidente, traductrice et lectrice silencieuse. Véritable Muse, lisant par-dessus l'épaule d'Abel, lui insufflant son énergie, et l'accompagnant finalement dans la vieille Cadillac rouge sang, avec de grands ailerons lumineux, pour aller cueillir Monsieur Melville dans l'ancien port de Harlem et le ramener dans le pays de la fiction québécoise.

Samm, après avoir eu sa propre cuvre, Discours de Samm (1983), revient dans Docteur Ferron. Puisqu'elle a trouvé dans son roman sa propre voix, elle devient même une narratrice de cet étonnant essai. Une des trois narrateurs se relayant la parole pendant ce pèlerinage à la mémoire de Jacques Ferron, les deux autres étant Abel, premier pèlerin, et Bélial. Cè même Bélial, diable boiteux, jadis prince des enfers, personnage protéiforme dans l'œuvre ferronienne et qui, à la fin de la Chaise du maréchal ferrant de Jacques Ferron, fut exilé à Montréal. Il est maintenant le chauffeur, le compagnon et le valet d'Abel, ayant troqué sa chaise volante pour une autre machine, une vieille Cadillac, blanche maintenant, mais toujours la même avec ses grands ailerons lumineux. 
Trois personnages romanesques, donc, dont l'un emprunté à l'œuvre ferronienne, qui partent ensemble pour parcourir les différents pays de Jacques Ferron afin de recomposer sa figure. Personnages à la recherche d'un auteur disparu. On aura compris que la structure est romanesque. Et non seulement parce que les conteurs sont des personnages fictifs. Ils se répondent les uns aux autres dans une configuration qui n'en reste pas moins délibérement romanesque par la fiction qui les détient, dira Victor-Lévy Beaulieü dans le «Liminaire» (p. 12), avant de leur céder définitivement la parole. Roman par sa structure narrative, cet essai l'est aussi parce qu'il est histoire d'amour, celle d'Abel et de Samm qui se retrouvent vingt ans après avoir pris rendez-vous, pour finalement devenir amants sous le ciel de Québec et habiter à la fin la maison de Trois-Pistoles. C'est grâce à la femme aimée, Muse devenue amante, que le pèlerin peut retrouver son père et renaître à la vie. Qu'il peut, en somme, assumer son premier amour, celui qui l'unit à son père.

Mais cet essai, surtout, participe du roman parce que la quête d'Abel, comme ce fut le cas pour les essais précédents, est tout autant quête de lui-même que celle de son objet. Á l'origine, il se voit confier une mission par une destinatrice, la femme de Jacques Ferron: retrouver l'authentique Jacques Ferron, en le démarquant des faux et en recomposant son être éclaté. Mission confiée dans un contexte cauchemardesque parce qu'Abel se sent coupable de ne pas l'avoir entreprise auparavant. Par négligence, parce qu'il a été happé par la vie et surtout par son travail de scripteur pour la télévision; par pudeur aussi sans doute, parce que malgré son impudicité congénitale, l'écrivain est conscient que le privé est sacré et qu'il devrait rester tel, même par-delà la mort (p. 392); par une hésitation et une crainte toutes naturelles, certainement, car cette quête aura son prix. Remise en question d'Abel lui-même en tant qu'écrivain, descente aux enfers, tentative de suicide, mort symbolique et, au bout de trois jours et de trois nuits de délire fiévreux, lente résurrection. Peut-être, même, naissance première plutôt que résurrection, car Abel, émergeant du néant comateux, aura l'impression de sortir d'entre les cuisses de [son] père (p. 408). Le fils ici, tout autant que dans la trinité divine, procède du père.

La remontée à la vie d'Abel, après sa mort symbolique, est aussi accession à la lucidité et à la pleine conscience. Conscience - qưi était celle de Jacques Ferron - que l'écriture est un moi crucifiant (p. 400), inséparable dé l'angoisse et de la désespérance. Grande leçon de choses, la plus belle sans doute que Jacques Ferron nous ait donnée. Car, malgré tout, il n'y a pas d'entreprise désespérée, il n'y a que des désespoirs entreprenants. (p. 408)

Ainsi, partir à la recherche du père présente certains dangers, même si cette recherche a pour fin putative de régénérer celui-ci. 
Mais s'agit-il vraiment pour Abel de régénérer ce père en littérature? $\hat{A}$ un certain niveau, certes. Victor-Lévy Beaulieu dit très clairement dans son "Liminaire" qu'il veut communiquer [sa] ferveur, [son] enthousiasme, [sa] reconnaissance et [son] affection pour. Jacques Ferron (p. 12). Cela est réel. Mais tout aussi réelle est la conscience, aiguë chez le père, sourde chez le fils, que l'avenir du fils ne peut consister qu'en une mise à mort du père. Victor-Lévy Beaulieu cite, à la fin de son "Liminaire*, une lettre de Jacques Ferron à John Grube, lettre fabuleusement ironique dans laquelle Jacques Ferron décrit Victor-Lévy Beaulieu en l'associant à Gdipe. Avec quelle allégresse il me culbuterait un jour de son chemin (p. 14), conclut-il. Et, racontant'sa première rencontre avec Jacques Ferron, Abel relate que celui-ci, dans une parodie de baptême, lui avait épinglé sur la chemise un macaron de Pierre Elliott Trudeau en disant: Mon fils, je te baptise au nom de Papa Boss, de son fils et de Pierre Elliott Trudeau. Pour les siècles des siècles, moi Ferron je te dis: ne sois jamais un tueur de pères. Amen. (p. 26)

Le mot est lâché. Car esti-on jamais sûr de ne pas avoir tué son père? Voilà peut-être l'aiguillon de ce pèlerinage. Qu'en est-il vraiment de la responsabilité d'Abel (de son incompréhension, de son impuissance, de sa simple présence comme nouveau maelström littéraire) dans la mort littéraire de Jacques Ferron qui, dix ans avant sa mort physique, aurait eu douloureusement conscience d'être un écrivain fini. Qui, néanmoins, continuait d'écrire. Et dé soumettre humblement ses manuscrits à son fils littéraire, maintenant son éditeur, ayant donc droit de vie ou de mort sur son cuvre. Renversement paradoxal des rôles, et fatidique. Car le fils éditeur publiera Rosaire, dernière cuvre de Ferron publiée de son vivant; mais étant donné l'indifférence de l'accueil de Rosaire, cette parution fut un.désastre (p. 394) et confirmera à Jacques Ferron qu'il était mort comme créateur et qu'il se survivait:

Le pèlèrin, en somme - et Abel en est sourdement conscient est aussi un rapace (faucon) et un prédateur redoutable (requin). Animal de proie. Comment départager les choses? Comment distinguer la dévotion de la dévoration ? Et c'est ainsi que tout pèlerin est un pénitent, sourdement conscient d'une responsabilité larvée. Voulant rendre hommage et faire acte de dévotion en visitant les lieux saintś mais tout autant motivé par le souci de faire œuvre de pénitence et d'expiation. Voyage souverainement complexe et ambigu, donc, et d'une fascination remarquable.

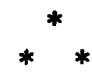

Face à cette quête d'une authenticité, d'une :vérité, d'une complicité qui transcende la mort, sommes-nous toujours dans le 
registre de l'essai? Nous sommes pour une bonne part dans la fiction, celle d'un romancier qui ne peùt guère sortir de son univers romanesque et pour qui la fiction pénètre tout. Mais nous sommes aussi et simultanément dans le domaine de l'essai, et dans l'univers de la critique littéraire, critique de pair, critique de créateur qui, même quand elle se fait narcissique, permet de connaître de l'intérieur l'objet de l'étude, Jacques Ferron, et de-le saisir dans son élan créateur. Critique qui est une des formes, peut-être la forme supérieure, de la création littéraire même.

Est-ce dire que seul Victor-Lévy Beaulieu y trouve son compte et que cet essai, comme fiction et comme critique créatrice, est son affaire et a donc peu à voir avec Jacques Ferron? Pas du tout. L'œuvre et la vie de Jacques Ferron sont au centre de cet essai. La riche érudition historique et ethnologique de Ferron, son talent de conteur, la luminosité de son style, l'ardeur de ses convictions, la complexité de ses passions et la profondeur de ses angoisses sont la matière même de ce livre. Pour avoir beaucoup fréquenté l'homme et l'œuvre et pour s'être senti son complice, son lecteur privilégié et, sans doute, son héritier spirituel, Victor-Lévy Beaulieu se sent habilité à parler de Jacques Ferron avec la plus haute autorité. Il le fait donc sous cette forme dialoguée, quelque peu fantaisiste, mais d'une rare efficacité.

Et même l'esprit rationaliste y trouve son compte. Car le pèlerin suit les traces de Jacques Ferron dans un cheminement chronologique bien ordonné. Le pays de Maskinongé, d'abord, puisque c'est le pays de l'enfance qui détermine l'individu par ses parents (p. 67); puis, après avoir escamoté l'adolescence (pour la raison étonnante que Jacques Ferron 'n'a jamais dit grand-chose sur la sienne [p. 67]), trajet à Rivière-Madeleine où commence la carrière du médecin et du conteur; retour à Montréal, rue Saint-Denis, en passant par l'étape de Trois-Pistoles; départ vers Québec; retour à Longueuil; virée à Moncton et point de chute final à Trois-Pistoles.

Ce parcours tortueux est peu écologique dans ses entrecroisements; mais une Cadillac fictive peut bien se le permettre, surtout avec un chauffeur infatigable. Le trajet s'impose, d'ailleurs, car Abel est incapable d'évoquer ailleurs qu'en Gaspésie la genèse du conteur; de lire et de commenter ailleurs qu'au château Frontenac le.Ciel de Québec; de remémorer ailleurs qu'à Montréal Ferron l'historien ou l'écrivain politique; d'aborder ailleurs qu'à Longueuil la crise d'octobre; d'évoquer ailleurs qu'à Cap-au-Marteau, devant un immense feu de grève, le théâtre de Jacques Ferron; de décrire ailleurs qu'à Moncton le drame des Roses sauvages. Chaque lieu fait surgir son cortège de personnages ferroniens. De plus, en roulant, ce sont de longs échanges entre les pèlerins et chacune des étapes permet de faire le point sur l'un ou l'autre aspect de ce polygraphe merveilleux que fut Ferron. 
Mais peut-être cet itinéraire en chassé-croisé, peu soucieux des reprises et indifférent à la distance ${ }^{2}$, n'est-il, après tout, qu'une image de la course du lecteur ou de la chasse au sens. Et moi que suis-je donc? Rien de plus qu'un lecteur perdu au centre d'un code chiffré et dont j'essaie de me tirer. (p. 169)

S'en tire-t-il? De tous les, faux Jacques Ferron qui sont là, faux parce que incomplets, a-t-il réussi à en faire venir un autre, l'authentique? (p. 19) Comment savoir? Puisque Victor-Lévy Beaulieu évoque l'exemple de la forme dialoguée de Monsieur Teste dans son "Liminaire ", on pourrait toujours lui rappeler ce que Valéry disait de l'impuissance de la biographie ou de l'histoire à atteindre autre chose que l'insignifiant. Ou encore, si l'on prétendait saisir la vérité de l'écrivain par l'autre bout, celui de l'œuvre, que là aussi, toujours selon Valéry, c'était peine perdue: l'œuvre renvoie à un masque, et le masque à la machine. On pourrait aussi s'étonner de la singularité ou du paradoxe d'un livre qui veut vénérer et rendre hommage à l'écrivain sans doute le plus irrévérencieux de la littérature québécoise. Mais à quoi cela servirait-il de le dire? Ce sont là vues de l'esprit. Qui ne résistent guère, d'ailleurs, à l'émotion ressentie à la lecture de cet essai exemplaire. Voici donc un Jacques Ferron, peutêtre l'authentique, mais en tout cas aussi réel, vivant et émouvant qu'on pourrait le souhaiter. Ce qui me porte à penser que de tous les romans de Victor-Lévy Beaulieu, ce sont ses essais qui sont les plus réussis.

1 Victor-Lévy Beaulieu, Docteur Ferron, pèlerinage, Montréal, Éditions internationales Alain Stanké, 1991, 417 p.

2 On s'étonne tout de même qu'avec un tel chauffeur, Abel ne se soit pas permis l'ouest du Canada (Ferron, médecin militaire y a sejourné) ou n'ait pas eu la tentation de se rendre a Varsovie (lieu d'un congres de psychiatres en 1973 et source de l'Exécution de Maski). 